\title{
THE C AND Q BANDING PATTERNS OF THE CHROMOSOMES OF LILIUM LONGIFLORUM (THUNB.)
}

\author{
by \\ PREBEN BACH HOLM \\ Department of Physiology, Carlsberg Laboratory \\ Gamle Carlsberg Vej 10 - DK-2500 Copenhagen, Valby
}

Key words: $C$ and $Q$ banding, centromeric, constitutive heterochromatin

\begin{abstract}
The morphology and the $\mathrm{C}$ and $\mathrm{Q}$ banding properties of the Lilium longiflorum karyotype have been investigated. Only $9 \mathrm{C}$ bands have been observed, while the $\mathrm{Q}$ bands occupy $40-50 \%$ of the chromosome volume. Some correlation has been observed between regions of higher Feulgen stainability and the $Q$ bands. The very low amount of centromeric $\mathrm{C}$ banding may reflect the absence of long stretches of highly repeated DNA. The relationship between the $\mathrm{C}$ and $\mathrm{Q}$ bands and constitutive heterochromatin is discussed in relation to the premeiotic and somatic interphase in Lilium longiflorum.
\end{abstract}

\section{INTRODUCTION}

Banding of chromosomes with quinacrine $(\mathrm{Q})$ and Giemsa staining following heavy $(\mathrm{C})$ or mild extraction $(G)$ has provided a major tool for the study of cytogenetic problems. The bands thus obtained are generally reproducible and identical for different somatic tissues $(5,7)$, allowing the use of such patterns in analysis of chromosome aberrations and also in evolutionary studies of eukaryotic karyotypes (24).

It is generally believed that banding patterns reflect the structural and compositional heterogeneity of chromosomes (24). The patterns may therefore provide insight into certain structural and functional properties of chromosomes during the mitotic cycle and the meiotic process.
Chromosome regions revealed by $\mathrm{C}$ banding generally correspond to constitutive heterochromatin being condensed during interphase and late replicating (48). Moreover, the few reports available on $\mathrm{C}$ banding in meiotic cells have demonstrated an identity with mitotic banding and also a persistence of $\mathrm{C}$ bands throughout meiotic prophase $(14,18)$. Furthermore, chiasmata have been reported to be absent from these regions (21).

Little conclusive evidence seems available on the nature of $\mathrm{G}$ and $\mathrm{Q}$ bands even though a variety of functions have been suggested (24). In meiosis there is evidence, that the somatic bands have their counterpart bands in pachytene bivalents $(20,25)$ as well as in diplotene, 
diakinesis chromosomes (6). Furthermore, chiasmata at diakinesis have been reported to be localized predominantly in interbands $(10,15)$.

The major purpose of this study was to provide a basis for relating the transient appearance of chromocenters during premeiotic interphase to the nature of the bands in metaphase chromosomes. The Lilium longiflorum chromosomes are of particular interest in this connection because their behaviour in meiocytes has been intensively studied (38). Moreover, observations of premeiotic interphase have revealed, that the heterochromatic blocks all show a late DNA replication and do not persist during meiotic prophase $(12,39)$.

The present paper is addressed to the details of the banding patterns in metaphase chromosomes in root tips of Lilium longiflorum as obtained by Feulgen staining and $\mathrm{C}$ and $\mathrm{Q}$ banding techniques. The outcome of this study has been to provide the necessary basis for analysing the nature of premeiotic chromocenter behaviour, and to emphasize some major differences in the banding patterns commonly studied in plants and animals.

\section{MATERIALS AND METHODS}

\subsection{Plants}

Actively growing root tips were obtained from a clone of Lilium longiflorum grown in the Royal College of Forestry phytotron in Stockholm. Some root tips were obtained from the same clone grown in a greenhouse at the Botanical Garden of Copenhagen. No differences were observed between the two groups.

\subsection{Pretreatment}

Five $\mathrm{mm}$ long root tips were excised, washed and placed in alufoil covered glass vials containing $0,05 \%$ colchicine for $5 \mathrm{hrs}$. at $20^{\circ} \mathrm{C}$.

\subsection{Fixation}

The material was fixed for $2 \mathrm{hrs}$. in 3:1 (absolute ethanol: glacial acetic acid), washed for 1 hour in three successive changes of $70 \%$ ethanol and if necessary stored in a refrigerator. Root tips for quinacrine staining were washed and stored in absolute ethanol.

\subsection{Feulgen staining}

The material was hydrolyzed for $8 \mathrm{~min}$. at $60^{\circ} \mathrm{C}$ in $1 \mathrm{~N} \mathrm{HCl}$, cooled in an icebath and stained with basic fuchsin for $1 \frac{1}{2}$ hours at room temperature. The apical $\mathrm{mm}$ of the root tips were macerated and squashed in $45 \%$ acetic acid on a microscope slide, frozen in a squash slide freezer and transferred to absolute ethanol after removal of the coverslip. The slides were dipped through a series of absolute ethanol and xylene and the squashes made permanent by mounting in seukittu.

\subsection{Quinacrine mustard}

The material was transferred from ethanol to $45 \%$ acetic acid, macerated, squashed, frozen, washed in absolute ethanol and hydrated through a graded ethanol-water series. After two 5 min. washes in Mcllvaine buffer pH 4,0, the material was stained for $20 \mathrm{~min}$. in a $50 \mu \mathrm{g} / \mathrm{ml}$ solution of quinacrine mustard (Sigma). The slides were subsequently washed three times in buffer for a total of $15 \mathrm{~min}$. and mounted in the same buffer (3). Preparations were analyzed with a Zeiss photomicroscope using incident illumination and photographed on Agfa Ortho 25.

\subsection{Giemsa C}

Satisfactory results have not been obtained with the procedure of ARRIGHI and HSU (1), but three other procedures proved satisfactory.

I) Frozen squashes were prepared as described and the slides dipped into absolute and 70\% ethanol and airdried. Alkaline extraction was carried out in a saturated solution of $\mathrm{Ba}(\mathrm{OH})_{2}$ at $60^{\circ} \mathrm{C}$ for $5 \mathrm{~min}$. After 5-10 min. washing in cold tap water and a brief wash in distilled water the slides were incubated for one hour in $2 x \operatorname{SSC}(0,3$ $\mathrm{M}$ sodium chloride $+0,03 \mathrm{M}$ trisodium citrate) at $60^{\circ} \mathrm{C}$. Staining was performed in Giemsa (R66, Gurr) diluted 50 fold with $1 / 15 \mathrm{M}$ phosphatebuffer $\mathrm{pH} 7,0$ (41).

II) The procedure was the same as I except that the alkaline extraction was carried out at room temperature (45).

III) Airdried preparations were treated with $0,01 \mathrm{M} \mathrm{Ba}(\mathrm{OH})_{2} .8 \mathrm{H}_{2} \mathrm{O}, \mathrm{pH} 12,3-12,5$ for 1,2 and 5 min., washed in tap water and distilled water, and incubated for up to $16 \mathrm{hrs}$. in $2 \times \mathrm{ssc}$ at 
$60^{\circ} \mathrm{C}$. Samples for Giemsa staining were taken every second hour.

\section{RESULTS}

The Feulgen stained karyotype of Lilium longiflorum $(\mathrm{n}=12)$ is shown in Figure la. SAT constrictions can be identified in the long arm of chromosome 3 and in the short arms of chromosomes 4 and 7 . The results are in agreement with the Lilium longiflorum karyotype established by STEWART (40). In addition to the major SAT constrictions several minor constrictions may be identified. Along the chromatid regularly spaced variations in the intensity of Feulgen staining can be seen.

In attempting to obtain a good and reproducible $\mathrm{C}$ band procedure, control of the extraction steps proved to be critical. The method of SUMNER (41) often gave excellent results, but nuclei and chromosomes were often overextracted and difficult to identify. The method of VOSA and MARCHI (45) often gave excellent differentiation and good preservation, but the products of alkaline extraction were not reproducible and frequently cells were either excessively or inadequately extracted despite the apparent constancy of conditions. The problems encountered in the first two banding procedures could be obviated by following procedure III, as detailed under Methods. Good differentiation was found with 10-12, 6-8 and 4-6 hours of incubation in $2 x$ SSC after 1, 2 and 5 min. extraction in $\mathrm{Ba}(\mathrm{OH})_{2}$. Alkaline and SSC treatments appear to act in compensatory fashion. Best results were obtained using the 5 min. $\mathrm{Ba}(\mathrm{OH})_{2}$ and 4-6 hours $2 x$ SSC combination, thus supporting earlier experience, that Lilium chromosomes are sensitive to prolonged incubation in hot SSC.

Although this modified $\mathrm{C}$ banding method is more effective than the others it is still not entirely reproducible. In general, spread chromosomes that are free of cytoplasm and cell wall give a more consistent response, but in order to obtain a reliable $\mathrm{C}$ band karyotype successive 2 hour samples were taken. Thus treated, the karyotype of Lilium longiflorum is very simple (Fig. $1 \mathrm{~b}$ ). With the possible exception of the band on chromosome 1, the remaining 8 bands seen in Figure lb have been identi- fied in all three Giemsa procedures. The bands are distributed as follows: 2 flank the SAT constriction in chromosome 3 ; one is distal to the SAT constriction in chromosome 7; none is present in chromosome 4; in chromosome 7 one is close to the telomere of the long arm and a second is proximal to a minor secondary constriction. The remaining three are localized at minor constrictions, distal on chromosome 8 and proximal on chromosomes 9 and 12. All these bands are reproducible with the possible exception of the distal one on chromosome 3 and no difference has been found between homologous chromosomes. The only clearly inconsistent feature of banding among the cells is the appearance of tiny bands or dots around or in the centromere region; their presence is erratic and appears to be unrelated to the staining of other bands.

The Lilium karyotype has been found to possess a very large number of $Q$ bands regularly spaced along the chromosomes (Fig. $1 \mathrm{c}$ ). The quality of the banding has been found to be dependent on the quality of the morphological preservation. If the chromosomes are improperly preserved the same bands can be identified, but with a lower intensity of fluorescence. In badly preserved specimens, no bands can be seen at all. If the interpretation presented above is correct, no evidence has been found for banding polymorphism. All $\mathrm{C}$ bands can be seen to be fluorescent except the $C$ band in the short arm of chromosome 7. Furthermore, centromeric $\mathrm{C}$ and $\mathrm{Q}$ banding is the same; the bands, if present, are small and form rarely. Only on chromosomes 5,7 and 10 do the relatively large centromeric $\mathrm{Q}$ bands occur somewhat more frequently.

A comparison of the $\mathrm{Q}$ banding pattern and the chromosome structure after Feulgen staining reveals some correlation between the $\mathrm{Q}$ bands and the chromomeres. Both are regularly distributed along the chromatids, often confluent with homologous regions in the sister chromatids and minor constrictions can be seen to be only weakly fluorescent. However, quantitation of the very large number of bands including subdivisions of these bands has not been successful. A correlation between bands and chromomeres has accordingly not been possible. 


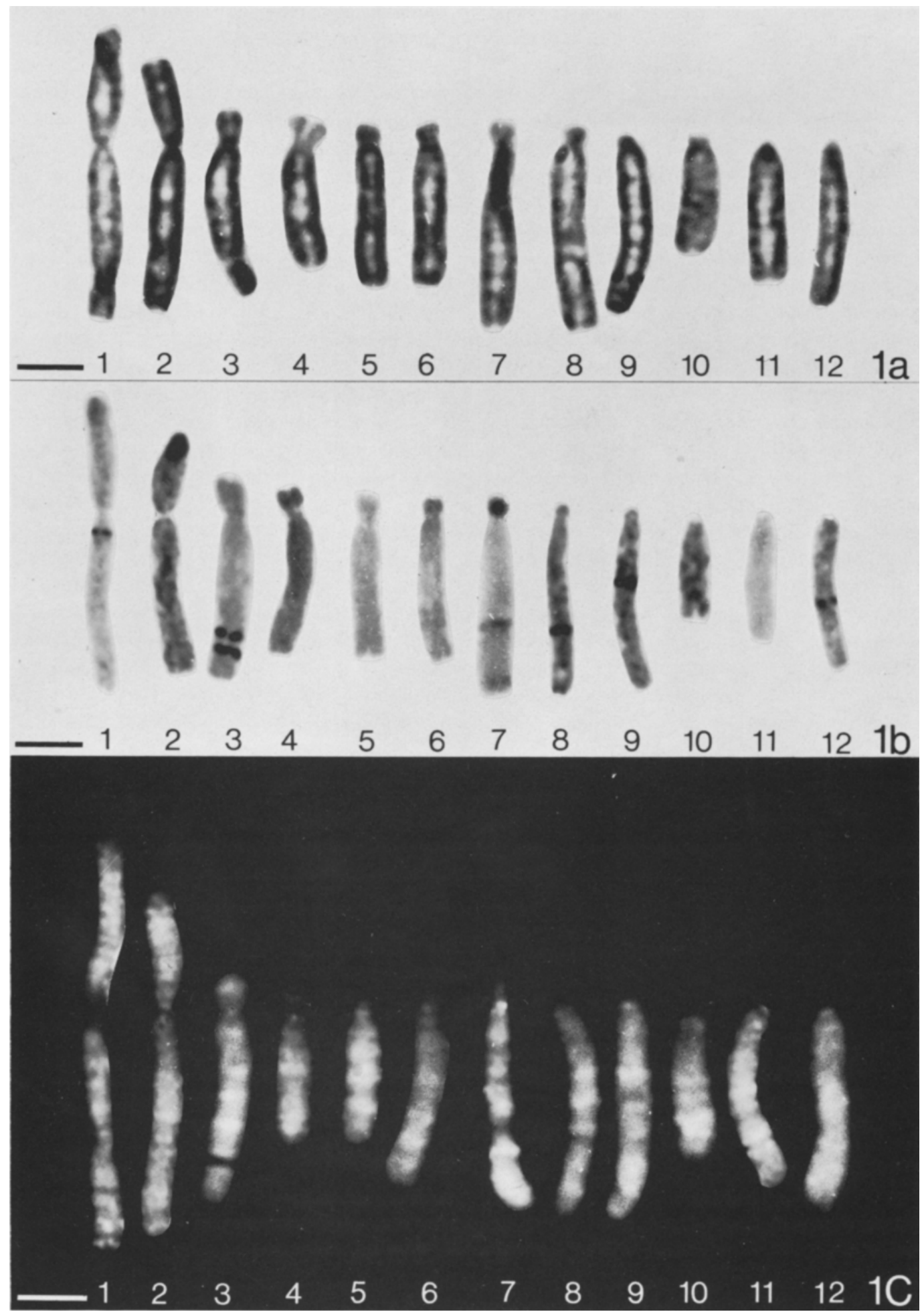

Figure 1. The karyotype of Lilium longiflorum. 1a) after Feulgen staining, 1b) after Giemsa C (III) staining, lc) after quinacrine murstard staining. $\times 2000$, $(\mathrm{bar}=5 \mu)$. 
An interpretation of the karyotype after $C$ and $Q$ banding is presented in the idiogram in Figure 2. Assuming an equal diameter of chromatids in banded and non banded regions, the bands constitute $39 \%$ of the chromosome volume; assuming the interbands take only $3 / 4$ of the band diameter, the band volume is $51 \%$. It should be stressed, however, that the boundary between bands and interbands is based on subjective criteria and to some extent is dependent on the photographic procedure. Finally, it seems quite likely that each band is subdivided into bands and interbands.

\section{DISCUSSION}

The nature of the $C$ bands is at present only partly understood. Studies of mammalian chromosomes especially have revealed that differential chromosome condensations, preferential extraction of DNA and protein in interband regions, and DNA and protein interactions all may be involved in the selective staining of the $\mathrm{C}$ band regions $(8,33)$. Moreover, in situ hybridization experiments have revealed that $\mathrm{C}$ bands are enriched in satellite DNA $(26,27)$.

The $\mathrm{C}$ banding pattern observed in the present study is very simple, all reproducible bands being confined to SAT and minor constrictions. Of particular interest is the virtual absence or very low amount of centromeric $\mathrm{C}$ bands, a trait that seems to be characteristic of most plants examined. Total absence of centromeric $\mathrm{C}$ bands has been reported in a variety of plants $(32,45)$. Re-examination of the same species and studies on other plants have demonstrated, however, that $\mathrm{C}$ bands occasionally show up in and around the centromere region $(2,19,22,36,42$, 44 ), suggesting that a minimum of centromeric constitutive heterochromatin is a universal part of the metaphase chromosome. STACK (36) suggested that $\mathrm{C}$ bands or dots in the centromere region actually were the kinetochores, defined as the region within the centromere to which the microtubules associate. Ross (30) suggested, however, that this staining pattern should be considered as a staining of centromeric heterochromatin. Larger centromeric $\mathrm{C}$ bands have only been observed in a few plants. Plantago ovata seems to possess quite large $C$ bands (37) correlating well with the classical constitutive heterochromatin observed by HydE (16). In Secale cereale the centromeric and telomeric $\mathrm{C}$ bands were observed to correlate well with classical heterochromatin (11).

At present, it seems difficult to evaluate to what extent these differences in $\mathrm{C}$ banding patterns are a reflection of natural quantitative and

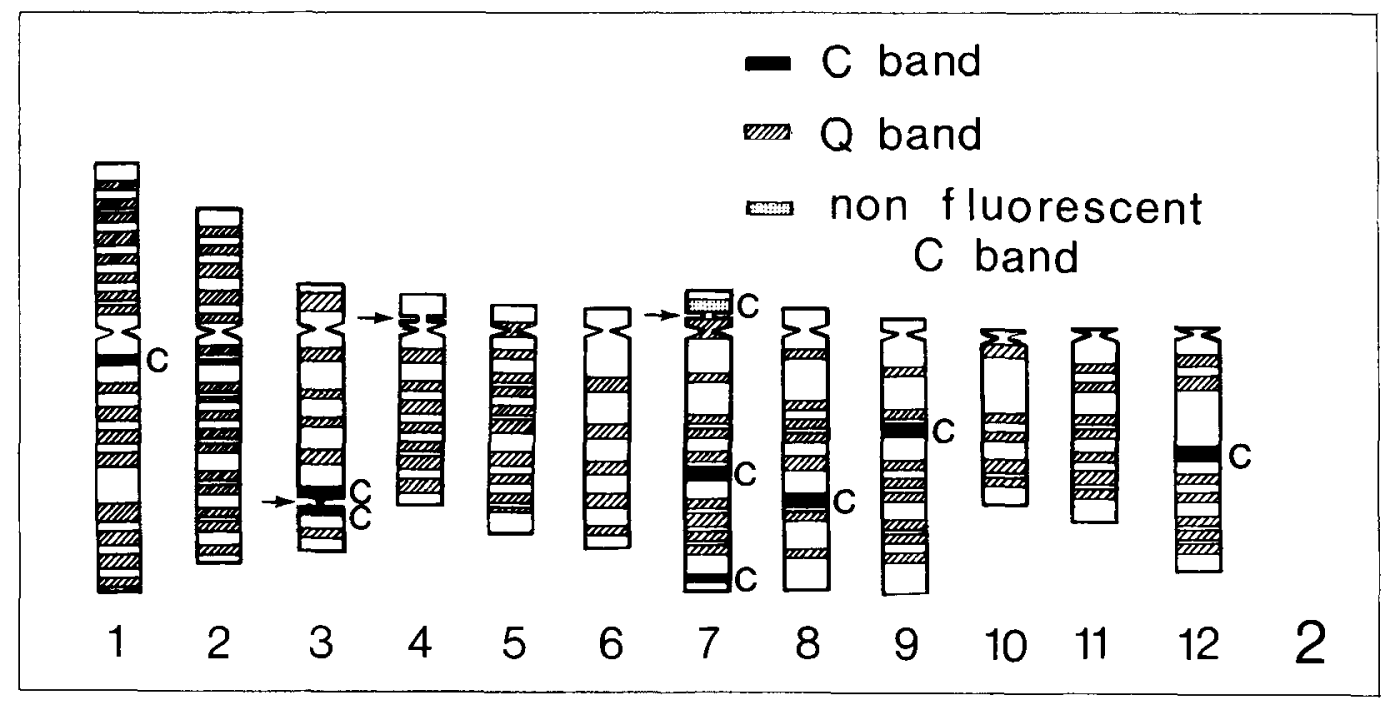

Figure 2. Idiogram of Lilium longiflorum, denoting $\mathrm{C}$ bands and $\mathrm{Q}$ bands. SAT constrictions are indicated with arrows. 
qualitative variations in chromosome structure between plants and animals. Proper $\mathrm{C}$ banding seems quite difficult to obtain in plants, and many reports suggest that different species require different treatments in order to obtain a reliable banding. Nevertheless the variation observed might reflect a variation in the amount of highly repetitive or satellite DNA. Interestingly many dicotyledons and all monocotyledons yet examined lack satellite DNA (17), even though repeated DNA sequences occur extensively (9). Likewise Lilium longiflorum does not possess any satellite DNA, but $80 \%$ consist of sequences repeated more than 10 times. Only $10 \%$ renaturates below a Cot value of $10,(13)$ and a correlation between the lack of very highly repeated DNA and the very low amount of centromeric heterochromatin could accordingly be possible. Very highly repeated DNA has been reported in other monocotyledons; in Secale cereale $10 \%$ renaturated with kinetics similiar to animal satellite DNA (28); in Triticum aestivum the amount was $4-10 \%$ and believed to be palindromic sequences (35).

Contrary to $\mathrm{C}$ banding, the $\mathrm{Q}$ banding study has revealed a highly complex system of $Q$ bands along the chromatids. The centromeric $Q$ bands seem to be as inconsistent as centromeric $C$ bands with the possible exception of the bands on chromosomes 5,7 and 10.

Reduced fluorescence in $\mathrm{C}$ bands has been noted in many organisms $(31,41)$ and has been suggested to be a quenching reaction due to high G-C content (46). VosA (43) furthermore described, that regions outside the $C$ bands could show reduced fluorescence. A similar phenomenon has been observed in the present study, but the regions are interpreted to represent minor constrictions. The extent to which a similar correlation between fluorescence intensity and Feulgen stainability exists in the rest of the Lilium longiflorum genome cannot yet be resolved because of the complexity of the band and chromomere pattern. It does suggest, however, that a primary reason for banding of chromosomes is variations in chromatin condensation along the chromatids as suggested by MCKAY (23) and by YUNIS and SANCHEZ (47). Photometric evidence for such a variation is still lacking (4) and, moreover, special treatments often seem necessary for obtaining a Feulgen banding comparable to $G$ and $Q$ bands (29). It seems at present quite clear that more information must be obtained on the native conformation of the metaphase chromosome and the effect of colchicine, fixation and posttreatments as well as the actual staining reaction before the nature of the bands can be elucidated.

At present, considerable confusion seems to exist on the definition of constitutive heterochromatin. Often all $\mathrm{C}, \mathrm{G}$ and $\mathrm{Q}$ bands are included in the definition, implying that the bands remain condensed during interphase and show a delayed DNA replication. Autoradiographical and cytological evidence from somatic and premeiotic interphase nuclei in Lilium longiflorum (12), suggest that $C$ bands fulfill this definition, while the behaviour of the $\mathrm{Q}$ bands is somewhat more complicated. Double staining of interphase nuclei with quinacrine mustard and orcein have demonstrated, that in many nuclei the majority of the chromocenters is fluorescent, but often the amount is as low as $50 \%$. Moreover, premeiotic interphase nuclei have been found to possess considerably more DNA packed into chromocenters than somatic interphase nuclei, altogether suggesting that $Q$ bands cannot be necessarily regarded as constitutive heterochromatin.

\section{ACKNOWLEDGEMENTS}

I am indebted to the late professor Mogens WESTERGAARD for suggesting this project and for his helpful guidance. I would also like to thank professor HERBERT STERN for valuable discussions and comments on the manuscript.

\section{REFERENCES}

1. Arrighi, F. E. \& T. C. Hsu. Localization of heterochromatin in human chromosomes. Cytogenetics 10: 81-86 (1971)

2. Bentzer, B. \& T. LANDSTrøm, Polymorphism in chromosomes of Leopoldia comosa (Liliacea) revealed by Giemsa staining. Hereditas 80 : 219 233 (1975)

3. Casperson, T., L. Zech, K. J. Modest, G. E. FOLEY, U. WAGH \& E. Simonson. Chemical differentiation with fluorescent alkylating agents in Vicia faba metaphase chromosomes. Exp.Cell. Res. 58: 12-140(1969) 
4. Casperson, L. Zech, E., J. Modest, G., E. Foley, U. WAGH, \& E. SIMONSON. DNA binding fluorochromes for the study of the organization of the metaphase nucleus. Exp. Cell. Res, 58: 141-152 (1969)

5. Casperson, T., G. Lomakka \& L. Zech. The 24 fluorescence patterns of human metaphase chromosomes, distinguishing characters and variability. Hereditas 67: 89-102 (1971)

6. Casperson, T., M. Hulten, J. Lindsten \& L. $\mathrm{ZECH}$. Identification of chromosome bivalents in human male meiosis by quinacrine mustard fluorescence analysis. Hereditas 67: 147-149 (1971)

7. Casperson, T., A. de la Chapelle, J. Schroder \& L. ZECH. Quinacrine fluorescence of metaphase chromosomes. Identical patterns in different tissues. Exp. Cell.Res. 72: 56-59 (1972)

8. Comings, D. E., E. Avelino, T. A. OKada \& H. E. WYANDT. The mechanisms of $\mathrm{C}$ and $\mathrm{G}$ banding of chromosomes. Exp. Cell. Res. 77: 469-493 (1973)

9. Flavell, R, B., M. D. Bennett, J. B. Smith \& D. B. SMith, Genome size and the proportion of repeated nucleotide sequence DNA in plants. Biochem.Genet. 12: 257-269 (1974)

10. Fox, D. P., C. Carter \& G. M. Hewitt. Giemsa banding and chiasma distribution in the desert locust. Heredity 31: 272-276 (1973)

11. Gill, B. S. \& G. Kimber. The Giemsa C-banded karyotype of rye. Proc.Nat.Acad.Sci. 71: 1247 . $1249(1971)$

12. HoLM, P. B. The premeiotic DNA replication of euchromatin and heterochromatin in Lilium longiflorum (Thunb.) In preparation

13. HOTTA, Y. \& H. STERN. Zygotene and pachytenelabeled sequences in the meiotic organization of chromosomes. In: The Eucaryotic Chromosome, W. S. PEacock \& R.D. Brock eds. Austral. Nat. Univ.Press Canberra pp. 283-300 (1975)

14. HSU, T. C., J. E. K. COOPER, M. L. MACE \& B. R. BRINKLEY. Arrangements of centromeres in mouse cells. Chromosoma (Berl.) 34: 73-87 (1971)

15. Hultén, M. Chiasma distribution at diakinesis in the normal human male. Hereditas $76: 55-78$ (1974)

16. HYDE, B. B. Differentiated chromosomes in Plantago ovata. Am.J.Bot. 40: 809-815 (1953)

17. Ingle, J., G. G. Pearson \& J. Sinclair. Species distribution and properties of nuclear satellite DNA in higher plants. Nature New Bio. 242: 193. $197(1983)$

18. Keezer, J. R. \& H. C. MACGregor. A fresh look at meiosis and centromeric heterochromatin in the red-backed salamander Plethodon cinereus cinereus (Green). Chromosoma (Berl.) 33: 146-166 (1971)

19. Klasterska, I. \& A. T. Natarajan. Distribution of heterochromatin in the chromosomes of Nigella damascena and Vicia faba. Hereditas 79: 154-156 (1975)
20. Luciani, J. M., M. R. Morrazzani \& A. STAHL. Identification of pachytene bivalents in human male meiosis using $G$ banding techniques. Chromosoma (Berl.) 52: 275-282 (1974)

21. Marks, G. E. Giemsa banding of meiotic chromosomes in Anemone blanda. Chromosoma (Berl.) 49: 113-119(1974)

22. Marks, G. E. \& D. Schweltzer. Giemsa banding: Karyotype differences in some species of Anemone and in Hepatica nobilis. Chromosoma (Berl.) 44: 405-416 (1974)

23. MCKAY, R. D. G. The mechanism of $C$ and $G$ banding in mammalian metaphase chromosomes. Chromosoma (Berl.) 44: 1-14 (1973)

24. Nobel Symposium 23. Chromosome identification-technique and application in biology and medicine. T. CASPERSON \& L. Zech, eds. Academic Press (New York and London) 355 p. (1973)

25. OKadA, T. A. \& D. E. Comings. Mechanisms of chromosome banding III. Similarity between G bands of mitotic chromosomes and chromomeres of meiotic chromosomes. Chromosoma (Berl.) 48: 65-71 (1974)

26. Pardue, M. L. \& J. G. Gall. Chromosomal localization of mouse satellite DNA. Science 168 : 1356-1358 (1970)

27. RAE, P. M. M. The distribution of repetitive DNA in chromosomes. Adv. Cell.Mol.Biol. 2: 109-149 (1972)

28. Ranjekar, P. K., J. G. Lafontaine \& D. PAllota. Characterization of repetitive DNA in rye (Secale cereale). Chromosoma (Berl.) 48: 427440 (1974)

29. Rodman, T. C. \& S. Tahiliani. The Feulgen banded karyotype of the mouse: Analysis of the mechanisms of banding. Chromosoma (B erl.) 42 : 37.56 (1973)

30. Ross, U. P. Are centromeric dots kinetochores? Nature 254: 463 (1975)

31. ROWLEY, J. D., W. F. BODMER. Relationship of centromeric heterochromatin to fluorescent banding patterns of metaphase chromosomes of the mouse. Nature 231: 503-506 (1971)

32. Sarma, N. P.\& A. T. Natarajan. Identifications of heterochromatic regions in the chromosomes of rye. Hereditas 74: 233-238 (1973)

33. SCHNEDL, W. Observations on the mechanisms of Giemsa staining methods. In: Nobel Symposium 23. Chromosome identification-technique and application in biology and medicine. $T$. CASPERSON \& L. ZECH, eds. Academic Press (New York and London) 1973

34. SCHWEItzer, D. Differential staining of plant chromosomes with Giemsa. Chromosoma (Berl.) 40: $307-320$ (I973)

35. SMith, D. B. \& R. B. Flavell. Characterization of the wheat genome by renaturation kinetics. Chromosoma (Berl.) 50: 223-242 (1975)

36. StaCK, S. M. Differential Giemsa staining of kinetochores and nucleolus organizer hetero- 
chromatin in mitotic chromosomes of higher plants. Chromosoma (B efl.) 47: 361-378 (1974)

37. StaCK, S. M. \& C. R. Clarke. Pericentric chromosome banding in higher plants. Canad.J.Genet.Cytol. 15: 367-369 (1973)

38. STERN, H. \& Y. HoTtA. Biochemical controls of meiosis. Ann.Rev. Genet. 7: 37-66 (1973)

39. Stern, H., M. WestergaARd, \& D. von WETTSTEIN. Presynaptic events in meiocytes of Lilium longiflorum and their relation to crossing over: A preselection hypothesis. Proc.Nat. Acad.Sci. 72: 961-965(1975)

40. STEWART, R. T. The morphology of somatic chromosomes in Lilium.Am.J. Bot. 34: 9-26 (1947)

41. SUmner, A. T. A simple technique for demonstrating centromeric heterochromatin. Exp. Cell. Res. 75: 304-306 (1972)

42. VosA, C. G. Heterochromatin recognition and analysis of chromosome variation in Scilla sibirica. Chromosoma (Berl.) 43: 269-278 (1973)

43. VosA, C. G. The entanced and reduced quinacrine fluorescence bands and their relationship to the Giemsa patterns in Allium flavum. In: Nobel Symposium 23. Chromosome identification technique and application in biology and medicine. T. CASPERSON \& L. ZECH eds. Academic Press (New York and London) pp. 156-158 (1973)

44. VosA, C. G. Heterochromatic patterns in Allium I. The relationship between the species of the cepa group and its allies. Heredity 36: 383-392 (1976)

45. Vosa, C. G. \& P. MarchI. Quinacrine fluorescence and Giemsa staining in plants. Nature New Biol. 237: 191-192 (1972)

46. Weisblum, B. \& P. Dehaseth. Quinacrine, a chromosome stain specific for deoxyadenylatedeoxythymidylate rich regions in DNA. Proc.Nat. Acad.Sci. 69: 629-632 (1972)

47. Yunis, J.J. \& O. Sanchez, G banding and chromosome structure. Chromosoma (Berl.) 44: $15-23$ (1973)

48. Yunis, J. J. \& W. G. Yasmineh. A model for mammalian constitutive heterochromatin. Adv. Cell.Mol.Biol. 2: 1-41 (1972) 